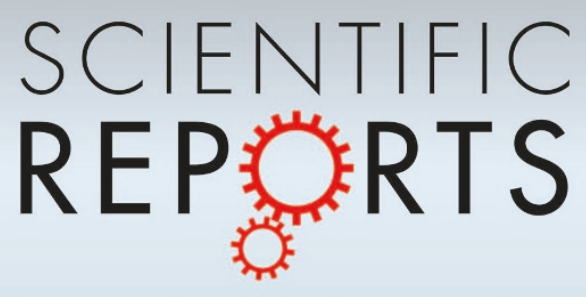

OPEN

SUBJECT AREAS:

DESIGN, SYNTHESIS AND

PROCESSING

TWO-DIMENSIONAL MATERIALS

TWO-DIMENSIONAL MATERIALS

CHEMICAL PHYSICS

Received

26 June 2013

Accepted

26 July 2013

Published

13 August 2013

Correspondence and requests for materials should be addressed to

X.J. (xin.jiang@unisiegen.de)

* These authors contributed equally to this work.

\section{When epitaxy meets plasma: a path to ordered nanosheets arrays}

\author{
Hao Zhuang ${ }^{1 *}$, Lei Zhang ${ }^{1,2 *}$, Regina Fuchs' ${ }^{1}$ Thorsten Staedler ${ }^{1} \&$ Xin Jiang ${ }^{1}$
}

${ }^{1}$ Institute of Materials Engineering, University of Siegen, Paul-Bonatz-Str. 9-1 1, 57076 Siegen, Germany, ${ }^{2}$ Peter Grünberg Institute and Ernst Ruska-Centre (ER-C) for Microscopy and Spectroscopy with Electrons, Forschungszentrum Jülich GmbH, 52425 Jülich, Germany.

The possibility of a controlled assembly of 2-dimensional (2D) nanosheets (NSs) into ordered arrays or even more sophisticated structures offers tremendous opportunities in the context of fabrication of a variety of NSs based devices. Reports of such ordered NSs are rare and all conventional "top-down" methods typically led to coarse structures exhibiting only limited surface quality. In this work, we demonstrate a path to directly synthesis ordered NSs arrays in a plasma activated chemical vapor deposition technique utilizing planar defects formed during hetero-epitaxial growth of crystals featuring a close-packed lattice. As an example, the synthesis of 3C-SiC NSs arrays with well-defined orientation on (001) and (111) Si substrates is shown. A detailed analysis identifies planar defects and the plasma environment as key factors determining the resulting 2D NSs arrays. Consequently, a "planar defects induced selective growth" effect is proposed to elucidate the corresponding growth mechanism.

$\mathrm{T}$ he unique optical, electronic and electrochemical properties of two dimensional (2D) nano-structured (nano-sheets or nano-plates) semiconductors ${ }^{1-5}$, metals ${ }^{6-8}$ and metal oxides ${ }^{9-11}$ qualify these structures as potent building blocks in numerous micro- or nano- electronic devices and, consequently, aroused strong interest in the scientific community. Key to unlock the full potential of all those structures is the ability to control their synthesis. In this context, synthetic approaches such as vapor transportation ${ }^{12}$, colloidal reaction ${ }^{8,13}$ and catalyst assisted chemical vapor deposition ${ }^{14}$ techniques have been successfully introduced. Yet, in order to fabricate 2D NSs based nano-devices, the ability to assemble NSs into arrays or more sophisticated structures represents a prerequisite. The requirements for large area devices such as catalyst support, sensors, photovoltaic cells, high surface area electrodes, efficient edge emitter for electron emission, and others are even more severe. Here, the NSs are required to "stand" in well-ordered arrays ${ }^{4,15-19}$. In contrast to the demand, reports of such ordered NSs arrays are rare. In this context, the bottleneck in processing ordered structures lies in the lack of efficient strategies to arrange the 2D structures once they have been synthesized. This step, however, is necessary as all of the above mentioned techniques result in a random distribution of NSs. An alternative approach, i.e. a conventional "top-down" etching strategy, is possible ${ }^{20}$. Unfortunately, the structures prepared by this path are typically coarse and feature only limited surface quality ${ }^{20,21}$, which hamper their performance ${ }^{21}$. Up to now, a feasible "bottom-up" approach, which would pose a solution to this dilemma, is lacking.

A large number of currently available 2D NSs features a close-packed arrangement of atoms (face centered cubic (FCC) structure, hexagonal close-packed (HCP) structure and their derivatives $)^{7,22-25}$. The reason for this lies not only in the fact that the majority of materials exhibits these crystallographic structures, but rather in the unique surface energy configuration of those arrangements making them the ideal candidates for the growth of NSs. For any close-packed arrangement, the surface energy of the close-packed facets is significantly lower compared to the other facets, which, in turn, leads to their diminished growth rates ${ }^{26}$. Such a difference in growth rate between close-packed and non-close-packed facets provides an inherent option for the formation of 2D NSs. Already in 1960, Hamilton and Seidensticker proposed a 2D growth model for FCC materials based on the general crystal growth theory ${ }^{27}$, which has been later on extended by others ${ }^{28-31}$. The model addressed the importance of planar defects (both twins and stacking faults) in accelerating the 2D growth of the close-packed planes to form nanoplates ${ }^{27}$. It has been widely applied to explain the growth of various FCC nanoplates in context of vapor- or liquid-phase synthesis in the past years ${ }^{32-35}$. Recently, a similar phenomenon has also been observed during the fabrication of nanoplates based on HCP lattices ${ }^{23,36}$. Due to their low formation energy, the presence of planar defects, i.e. twins and stacking faults, is a universal phenomenon while synthesizing materials with closepacked structures. Particularly in case of hetero-epitaxial film growth, a high density of uniformly distributed planar defects is present at the film/substrate interface. These are aligned parallel to the $\{111\}$ and $\{0001\}$ facets for 
FCC and HCP structures, respectively. Although the formation of these planar defects offers a mechanism to release the stresses induced by the large lattice mismatch between substrate and film, typically one strives to avoid them in the interest of obtaining perfect crystals. Aiming for an ordered growth of NSs arrays, however, the existence of these well-orientated planar defects might serve as favorable sites for NS growth following the suggestions of Hamilton and Seidensticker's model. Moreover, a controlled incorporation of planar defects in any NSs potentially opens up the opportunity to modify the NSs' electronic structure at the nanoscale, i.e. band gap, carrier density, and other. This would allow for a possibility to tailor the intrinsic properties of $\mathrm{NSs}^{37,38}$. Even though the approach seems potentially rewarding and any success would most likely have dramatic impact on new generations of nano-electronic devices, up to now, no attempt has been reported that deliberately took advantage of such planar defects in context of the controlled fabrication of NSs arrays. In this study, using the example of 3C-SiC NSs arrays, we present a generally valid approach to reliably initiate the extension of planar defects during hetero-epitaxial growth and, in turn, fabricate well-ordered 2D NSs arrays. In the particular case, presented here, the strategy is demonstrated for (001) and (111) Si substrates in combination with a microwave plasma chemical vapor deposition (MWCVD) process.

\section{Results}

To fabricate the 3C-SiC NSs arrays, we use tetramethylsilane (TMS) as single source precursor. High power $(2500 \mathrm{~W})$ microwave plasma is generated to activate the gas phase reaction. During fabrication, the TMS is diluted in $\mathrm{H}_{2}$; a very low concentration (140 ppm) corresponds to a low growth rate $(\sim 50 \mathrm{~nm} / \mathrm{h})$ of the $3 \mathrm{C}$-SiC epitaxial layer (see Supplementary Information for details). Typically the highest density of planar defects is observed at the 3C-SiC/Si interface. An increase of layer thickness, however, results in a reduction of the density of planar defects as they start to annihilate each other ${ }^{39,40}$. Here, the low growth rate of the $3 \mathrm{C}-\mathrm{SiC}$ epitaxial layer ensures a low annihilation rate and consequently offers sufficient time for the formation of 2D NSs.

The successful synthesis of NSs arrays is confirmed by scanning electron microscope (SEM). Figures 1a,b show the SEM images of the surface morphology of the NSs on (001) and (111) Si substrates, respectively. In both cases, the NSs feature a width of 1-2 $\mu \mathrm{m}$ and show a uniform distribution over the wafer surfaces. A simple tracking of the number of the NSs per SEM image results in a density of the NSs. These are approximated to be $3.7 \times 10^{7} / \mathrm{cm}^{2}$ and $3.4 \times 10^{7} /$ $\mathrm{cm}^{2}$ on the (001) and (111) Si wafers, respectively. In order to determine the structure of the NSs, selective area electron diffraction (SAED) was carried out on a single NS in a transmission electron microscope (TEM, Supplementary Fig. S2). The SAED pattern can clearly be indexed based on the structure of $3 \mathrm{C}-\mathrm{SiC}$ as it showed the characteristic pattern of $3 \mathrm{C}-\mathrm{SiC}$ along the [011] direction featuring a lattice constant of $0.43 \mathrm{~nm}$. Furthermore, Fig. 1a,b reveals a textured nature of the NSs: On a (001) Si substrate the NSs show an angle of $90^{\circ}$ with respect to each other, while they exhibit an angle of $120^{\circ}$ for the (111) Si substrate. These angles are in good agreement with the ones formed between the $<011>$ directions, i.e. $90^{\circ}$ and $120^{\circ}$ on the (001) and the (111) facets, respectively. Additional XRD investigation is able to show a cubic-on-cubic orientation relation between the $3 \mathrm{C}-\mathrm{SiC}$ NSs and the underlying Si substrates (Supplementary Fig. S3). This phenomenon provides evidence for an epitaxial growth of the 3C-SiC NSs on the Si substrate.

Figures 1c,d show the SEM cross-sectional images of the NSs along the $\langle 110\rangle$ direction of the Si substrates. From the images it is possible to identify an intermediate layer between the NSs and the substrates. This layer acts as the "seeding layer" for the nucleation and growth of NSs. More detailed information of its function will be given in the "Discussion" part. Moreover, all NSs are tilted. NSs on
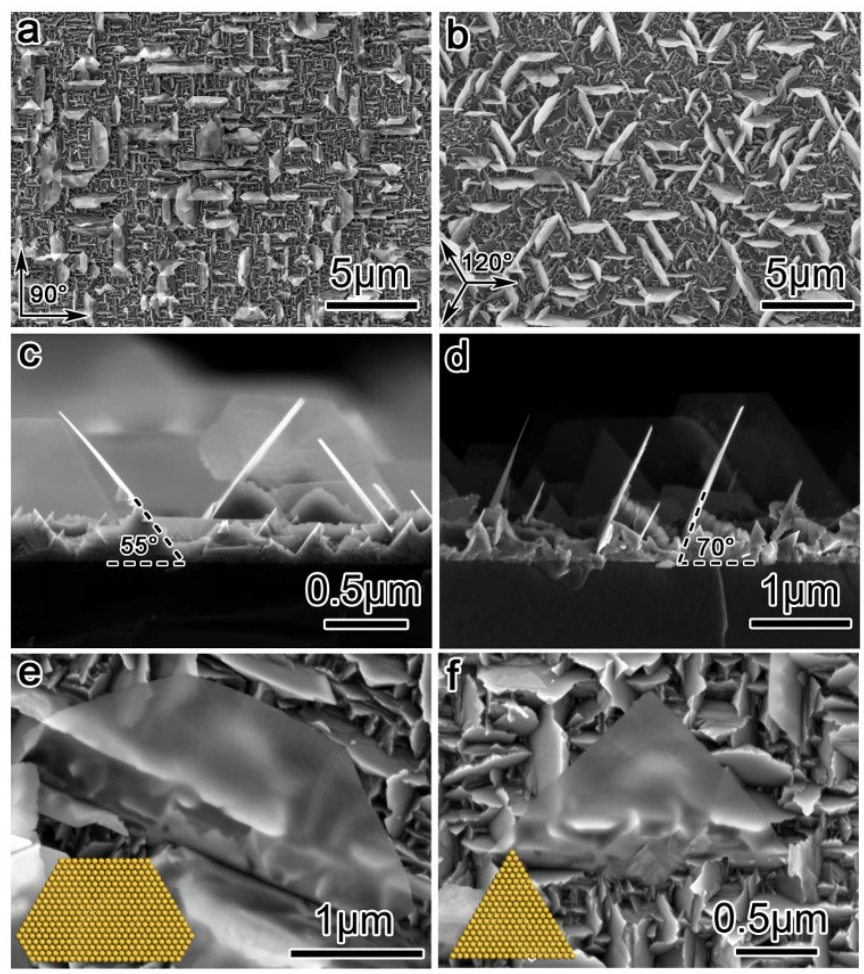

Figure 1 | SEM images of the 3C-SiC NSs arrays grown on (001) and (111) Si substrates. (a) Surface image of NSs on (001) Si; (b) surface image of NSs on (111) Si; (c) cross-sectional image of NSs on (001) Si; (d) crosssectional image of NSs on (111) Si; (e) and (f) high resolution SEM surface images of one $3 \mathrm{C}$-SiC NS, inset shows the restored shape of the corresponding NS.

the (001) Si substrate form an angle of $55^{\circ}$ with the substrate surface, while the ones on the (111) Si feature an angle of $70^{\circ}$, respectively. Both are characteristic angles in a FCC lattice: $55^{\circ}$ represents the angle between $\{001\}$ and $\{111\}$ facets and $70^{\circ}$ the one between $\{111\}$ facets. In combination with the parallel orientation of the $3 \mathrm{C}-\mathrm{SiC}$ NSs with respect to the Si substrates these angles indicate that the basal plane of the NSs is composed of $\{111\}$ atomic layers. This conclusion is further strengthened by an analysis of the shape of the NSs. As NSs on both substrates feature the same crystallographic character, the following consideration is only carried out for the structures synthesized on the (001) Si substrate.

Figures 1e,f show high-resolution SEM images of two typical shapes of the NSs. It is worthwhile to note, that these images only represent the projections of the structures onto the (100) plane and, therefore, include a corresponding distortion with respect to their actual shape. These actual or "true" shapes of the NSs, which are shown in the insets of Fig. 1e,f, can be easily restored taking the tilt of $55^{\circ}$ into account. The hexagonal shape (interior angles: $120^{\circ}$ ) as well as the equilateral triangular shape with edges along the $\langle 110\rangle$ directions both confirming the $\{111\}$ orientation of the basal plane of the NSs. This final observation agrees well with our prediction discussed previously: the $\{111\}$ facets show the slowest growth rate among all the facets as they feature the lowest surface free energy. This, in turn, leads to the growth of crystals exhibiting a large $\{111\}$ surface. One final, additional, conclusion can be drawn from the SEM observations. Figures 1e,f clearly show that the corresponding NSs are electron transparent as one can make out the detailed morphology underneath. This typically is only possible for ultra-thin nano-structures and allows for an estimation of the thickness of our NSs by calculating the electron penetration depth at the given imaging conditions (See Supplementary Information for details) ${ }^{7}$. As 
depicted in Supplementary Fig. S4, the NSs in the present study will show transparency for electrons in an energy range of $0.5-1 \mathrm{keV}$, corresponding to an upper limit of their thickness on the order of $6-$ $20 \mathrm{~nm}$.

Detailed TEM analysis shown in Fig. 2 reveals further crystallographic and morphological information of the NSs. Figure 2a represents a bright field cross-sectional overview of a NS. The corresponding SAED pattern at the $\mathrm{SiC} / \mathrm{Si}$ interface is shown in Fig. $2 \mathrm{~b}$. Because of the application of glue to the surface of the samples during the TEM cross-section sample preparation process, a slight bending of the NS is observed in Fig. 2a. Nevertheless, this has no effect on the conclusions we draw in the present study. In the SAED pattern, discrete diffraction spots of both $\mathrm{Si}$ and $3 \mathrm{C}-\mathrm{SiC}$, respectively, can be observed. The good match between the spots corresponding to $3 \mathrm{C}-\mathrm{SiC}$ as well as $\mathrm{Si}$ substrate confirms the epitaxial growth nature of $3 \mathrm{C}-\mathrm{SiC}$ on $\mathrm{Si}$. The weak streaks, which are noticeable between the diffraction spots of $3 \mathrm{C}-\mathrm{SiC}$, indicate the existence of planar defects. The direction of the streaks even allows to conclude that all planar defects are aligned parallel to the $\{111\}$ planes. Magnified TEM images (Fig. 2c-e) confirm the existence of planar defects throughout the NS. Even though the upper part of the NS is ultra-thin (Fig. 2c), it was still possible to observe a twin structure (see the inset). Actually, this twin boundary originates at the bottom of the NS penetrating through the whole NS. This observation agrees well with Hamilton and Seidensticker's growth model discussed above: planar defects accelerate the $2 \mathrm{D}$ growth of the close-packed planes. The thickness of the NS is estimated to be $\sim 5 \mathrm{~nm}$ at the tip (Fig. $2 \mathrm{c}$ ) and $\sim 20 \mathrm{~nm}$ at the position just above the intermediate layer between substrate and NS. This is in accordance with the previous estimation of the thickness based on electron transparency $(6-20 \mathrm{~nm})$.
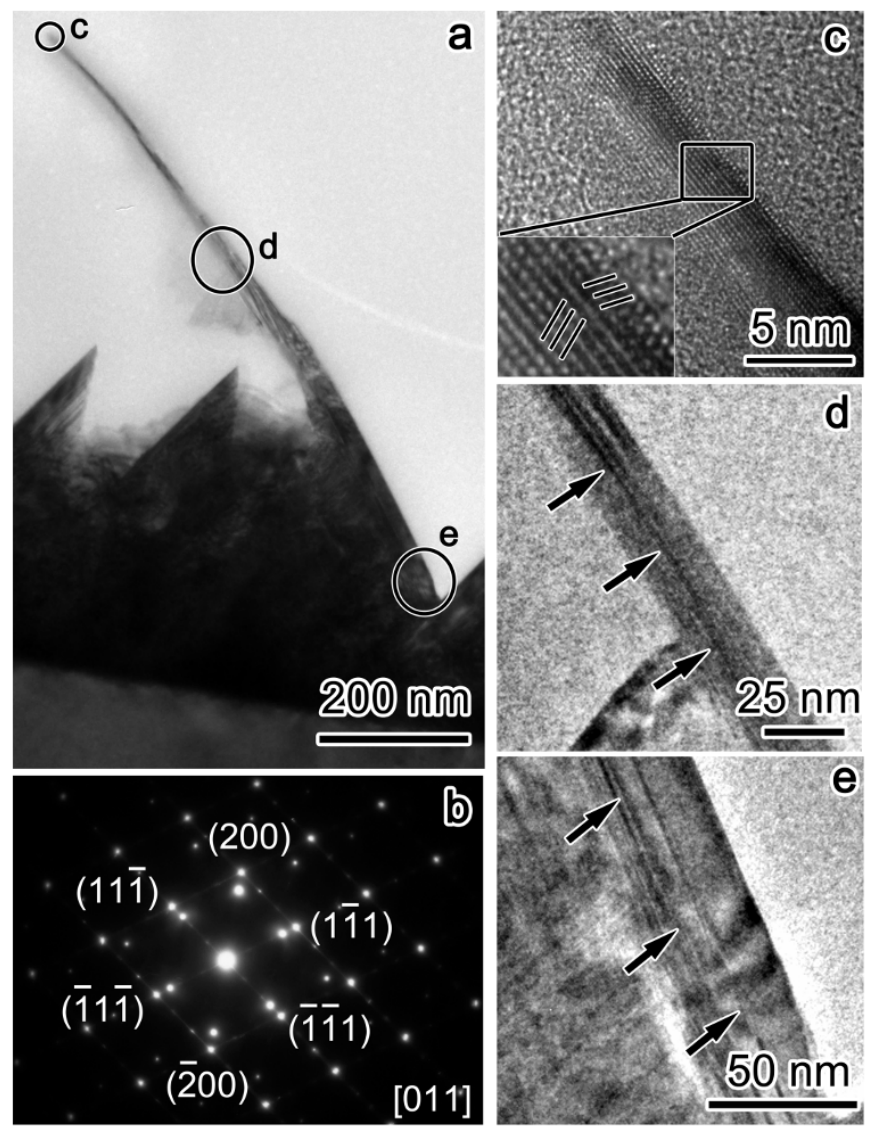

Figure $2 \mid$ TEM cross-sectional image of one NS. (a) overview, (b) SEAD at the $3 \mathrm{C}-\mathrm{SiC} / \mathrm{Si}$ interface of (a), (c-e) high magnification images of selected regions from (a).

\section{Discussion}

All of the results mentioned so far confirm the successful synthesis of well-ordered thin 3C-SiC NSs arrays by the extension of planar defects during the hetero-epitaxial growth of $3 \mathrm{C}-\mathrm{SiC}$ in a MWCVD process. In order to improve the control over size and density of our structures and at the same time to show the general value of our approach for other closed-packed structures we will spend the following paragraphs on shedding some light on the growth mechanism itself. To pursue this issue, different stages of the growth were studied. After $1 \mathrm{~h}$ deposition, a thin film without NSs forms (Fig. 3a). This film shows a mosaic-like morphology, which is typical for epitaxial $3 \mathrm{C}-\mathrm{SiC}$ films grown on an on-axis $\mathrm{Si}$ wafer ${ }^{41}$. A detailed look at the cross-section reveals the serratedshape of the film surface (Fig. 3b). Here, the low growth rate of the $\{111\}$ facets of $3 \mathrm{C}-\mathrm{SiC}$ lead to a gradual expansion of the $\{111\}$ facets, which, in turn, form sharp edges of the crystal ${ }^{41}$. The peak-to-valley height of the surface is determined by AFM measurement (Fig. 3c). The resulting $\sim 60 \mathrm{~nm}$ are quite remarkable for a film of only $\sim 80 \mathrm{~nm}$ thickness. This layer is referred to as "seeding layer", as it provides a large number of planar defects for the future growth of NSs. Figures 3d,e show SEM images of NSs grown for $2 \mathrm{~h}$ and $6 \mathrm{~h}$, respectively. It can be observed that small NSs already form after $2 \mathrm{~h}$ deposition and the size of the NSs continues to expand with increasing deposition time up to $6 \mathrm{~h}$. In contrast to this, the thickness of the "seeding layer" hardly increases at all, as shown in the insets of Fig. 3d,e, respectively.

Based on these findings, it can be easily inferred that the formation of NSs requires an epitaxial 3C-SiC "seeding layer" with sufficient roughness. Over deposition time, their size expands (from $\sim 1 \mu \mathrm{m}$ in width after 2 h growth to more than $5 \mu \mathrm{m}$ in width after 6 h growth); whereas the thickness of the "seeding layer" remains unchanged (it is $\sim 100 \mathrm{~nm}$ after $2 \mathrm{~h}$ growth and still remains $\sim 100 \mathrm{~nm}$ after $6 \mathrm{~h}$ growth). To elucidate the growth mechanism of the NSs further, it
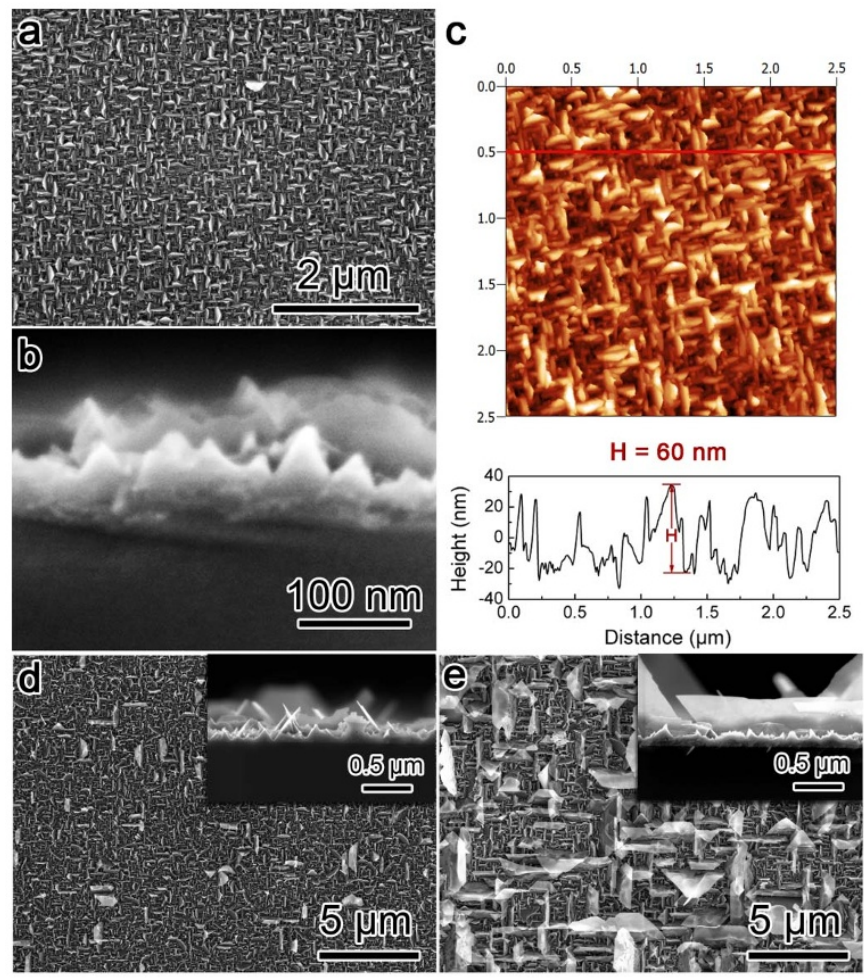

Figure 3 SEM and AFM images of the 3C-SiC NS with different growth times. (a) 1 hour growth; (b) cross-sectional image of (a); (c) AFM surface topography of (a) and its height profile; (d) 2 hours growth, (e) 6 hours growth. 
is essential to understand the factors, which trigger the initial formation of the NSs on the "seeding layer" and guarantee its continuous growth. According to Levchenko et al.'s investigation, the development of nanostructures in the plasma CVD process depends on the ion current distribution ${ }^{42}$. These ions are mainly accelerated in the sheath region between the plasma and the substrate. The sheath thickness, which, for the present study, is on the same length scale as the Debye length ${ }^{42}$, plays an important role in determining the ion current. In our work, the Debye length turns out to be less than $8.5 \mu \mathrm{m}$ (see Supplementary Information for details), which results in a very thin sheath thickness. Consequently, the topography and conductivity of any surface structures have a significant influence on the ions flux from the plasma to the substrate ${ }^{42}$. According to these considerations, once the ratio between nanostructure height and sheath thickness is large enough, the reactive ions in the plasma will be either attracted to or deflected by the corresponding nanostructures for conductive or insulating structures, respectively ${ }^{42}$. In the present study, both, the $3 \mathrm{C}-\mathrm{SiC}$ "seeding layer" as well as the $3 \mathrm{C}$ $\mathrm{SiC}$ NSs should show a good conductivity as they feature a large number of planar defects. The latter, according to Tsuboi et al.'s calculation, results in an extremely high electrical conductivity (of the order of $\left.10^{2} \mathrm{~S} / \mathrm{cm}\right)^{43}$. This assumption is confirmed by Song et al.'s experimental observation. In their work, they were able to show that the electric activity of such defects is even higher than that of the heavily nitrogen doped $\left(5 \times 10^{18} \mathrm{~cm}^{-3}\right) 3 \mathrm{C}-\mathrm{SiC}^{44}$. Therefore, even though they are not intentionally doped, our NSs, owning to their high density of defect, are highly conductive (see the corresponding electrochemical results in Supplementary Fig. S5 for confirmation). The consequence for our synthesis is obvious: as soon as the conductive sharp edged $3 \mathrm{C}$-SiC crystals reach a critical height in the growth process, the reactive ions in the plasma will be attracted to their corresponding outer edges. Levchenko et al. state a critical crystal height of $200 \mathrm{~nm}$ at a Debye length of $52 \mu \mathrm{m}^{42}$. Considering the significantly shorter Debye length $(<8.5 \mu \mathrm{m})$ in the present study, a peak-to-valley height of $\sim 60 \mathrm{~nm}$ seems reasonable to initiate the mechanism mentioned above. This mechanism results in a high concentration of reactive ions in the vicinity of the outer edges, which promotes a locally increased growth rate. Therefore, any "suitable" planar defects at these outer edges favor an accelerated growth of NSs along the defects.

Nevertheless, not all crystals featuring a sharp top edge necessarily evolve into NSs (Fig. 3e). In order to identify the necessary configuration of planar defects that favor a NSs growth, a detailed TEM analysis of "seeding layer" is carried out. Figures 4a,b represent TEM cross-sectional images of two characteristic crystal shapes in the seeding layer, which have not yet evolved into NSs. In Figure 4a, a crystal is observed for which the planar defects crisscross and consequently annihilate each other. At this point it is worth mentioning that the accelerated growth along the planar defects requires the formation of sub-steps at their side-faces, as these sub-steps significantly facilitate the incorporation of the reactive ions $\mathrm{s}^{27}$. The annihilation of defects, however, inhibits the formation of such substeps resulting in perfectly smooth $\{111\}$ side planes. Therefore, even though such crystals still attract the reactive species from the plasma, apparently, no accelerated growth along the planar defects occurs and the crystal is only able to expand relatively slowly by means of a layer-by-layer growth. The crystal shown in Fig. $4 \mathrm{~b}$ features a number of parallel planar defects without any crisscrossing. These planar defects create a corresponding number of sub-steps at the side of the crystal, which, in turn, favor an accelerated growth along the defects. Due to a decreasing concentration of reactive ions from the outer edge of the crystal to its bottom, the corresponding growth rate, however, will decrease accordingly. This trend is indicated by arrows in Fig. 4b. Such growth would not result in any NSs but would lead to the formation of crystals featuring a triangle shaped cross-section.

Based on the above discussion, the synthesis of NSs is successful only on the very thin layer of the planar defects that are exposed to the reactive ions. Figure $4 \mathrm{c}$ shows such configuration of planar defects, which meets this requirement. In this case, it is possible to observe the formation of a NS. Here, only a planar defect layer featuring a thickness of about $10 \mathrm{~nm}$ is present at the top of the crystal exposing the side-faces of the defects to the reactive species. All other defects, even though they are present within the crystal, annihilate by crisscrossing with the top ones and consequently exhibit no sub-steps at the side face. The NS growth is only realized by reactive species, which prefer to bond to the sub-steps of the thin defect layer at the top. Here, the high local concentration of reactive ions guarantees the supply of enough reactive ions for a continuous growth. As a consequence, small NSs start to form. Once the small NSs have formed, the second phase of growth is activated. During this phase the NSs increase in height and size, which results in a stronger attraction of reactive ions from the plasma to their edge. With more reactive ions being attracted by the NSs the number of ions being able to reach the "seeding layer" decreases, resulting in a decreasing growth rate of this layer (Fig. 3d,e). Finally, we have been able to identify the plasma environment as well as the planar defects as the key factors in the synthesis of 3C-SiC NSs arrays. On the one hand, the plasma environment generates reactive ions, which are sensitive to the substrate conditions. On the other hand, the planar defects in the NSs guarantee the continuous growth of these structures. Here, the effects of the defects are in two ways: (a) they create sub-steps on the side-faces of the NSs which are required for the preferential bonding of the reactive ions, and (b) they ensure the good conductivity of the NSs, which continuously attract reactive ions to accelerate the growth. Consequently we would like to refer to this mechanism as "planar defects induced selective growth" effect.

In summary, well-ordered 3C-SiC NSs arrays on (001) as well as (111) Si wafers have been prepared by means of a planar defect "catalyzed" growth in a MWCVD environment. The plasma process is utilized to initiate the extension of planar defects during the heteroepitaxial growth of 3C-SiC. XRD and TEM analyses show evidence for an epitaxial growth of the NSs on the Si wafers. The thickness of
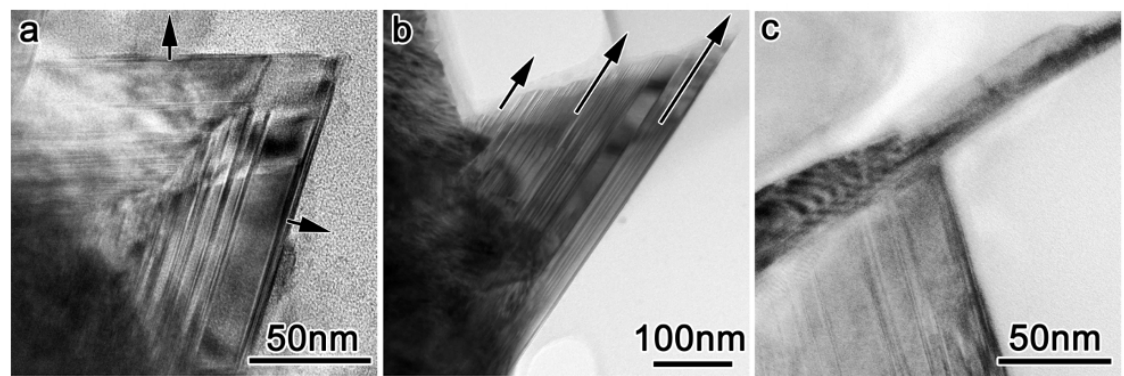

Figure $4 \mid$ TEM cross-sectional images of different crystals in the seeding layer. (a) and (b) crystals which have not yet evolved into NSs; (c) crystal which triggers the formation of a NS. The arrows represent the growth direction of the crystals. The length of the arrows qualitatively represents the growth rate at the corresponding position. 
the NSs is estimated to be 6-20 $\mathrm{nm}$. The NSs are uniformly distributed on the surface featuring a density of $\sim 3.7 \times 10^{7} / \mathrm{cm}^{2}$ and $3.4 \times$ $10^{7} / \mathrm{cm}^{2}$ on (001) Si and (111) $\mathrm{Si}$, respectively. Planar defects are observed throughout the NSs. These are particularly required to attract reactive ions to the edge of the NSs in order to accelerate and sustain their growth. A "planar defects induced selective growth" effect is proposed to elucidate the growth mechanism of the NSs. From this model, it would be possible to control the density of the NSs in the future. To decrease the density of the 3C-SiC NSs, the density of the planar defects on the surface should be decreased. This can be achieved by using undulant $\mathrm{Si}$ as substrate which is well described by Nagasawa et al. ${ }^{41}$ or to grow a thicker hetero-epitaxial "seeding layer" before starting the growth of 3C-SiC NSs (thicker epitaxial layer has lower defect density because of the annihilation of the planar defects). To increase the density of the 3C-SiC NSs, we should increase the possibility of the extension of the planar defects. This can be possibly done by increasing the roughness of the substrate by etching so that the "critical" peak-to-valley height of the seeding layer can be earlier achieved. It is important to point out, that, owning to the widespread use of the plasma enhanced CVD technique in the synthesis of various materials featuring a closepacked structure ${ }^{45-48}$, the approach shown here is not only limited to the fabrication of $3 \mathrm{C}-\mathrm{SiC}$ NSs arrays, but also applicable to other materials such as diamond ${ }^{45}, \mathrm{Si}^{46}, \mathrm{ZnO}^{47}$, and cubic $\mathrm{BN}^{48}$ etc. The $3 \mathrm{C}$ $\mathrm{SiC}$ NSs obtained in the present study are of great scientific importance on their own. Theoretical predictions exists which attribute very unique properties to $3 \mathrm{C}-\mathrm{SiC} \mathrm{NSs}{ }^{49-52}$, such as half-metallicity ${ }^{49}$, termination dependent ferro- or antiferromagnetic behavior ${ }^{50}$, transformation from indirect to direct band gap $^{51,52}$, and others.

\section{Methods}

Single-crystalline p-type $\mathrm{Si}(100)$ and (111) wafers were utilized as substrates for the growth of 3C-SiC NSs. The reactive gas featured a mixture of $\mathrm{H}_{2}$ and $\mathrm{Si}\left(\mathrm{CH}_{3}\right)_{4}$ [tetramethylsilane (TMS)] with a total flow rate of $400 \mathrm{sccm}$ (cubic centimeter per minute at STP). For the depositions of 3C-SiC NSs, a TMS concentration of $140 \mathrm{ppm}$ is used. During depositions, the Si substrate was firstly pretreated in pure $\mathrm{H}_{2}$ plasma for $5 \mathrm{~min}$ at a microwave power of $2200 \mathrm{~W}$, a gas pressure of $55 \mathrm{Torr}$, and a substrate temperature of $800^{\circ} \mathrm{C}$. The goal of this pretreatment was to remove the thin natural $\mathrm{SiO}_{2}$ surface layer. Subsequently, TMS was introduced to the gas phase in order to initiate the $\mathrm{SiC} \mathrm{NSs} \mathrm{growth.} \mathrm{At} \mathrm{the} \mathrm{same} \mathrm{time,} \mathrm{the} \mathrm{microwave} \mathrm{power,} \mathrm{gas} \mathrm{pressure} \mathrm{and}$ substrate temperature have been increased to $2500 \mathrm{~W}, 65$ Torr and $950^{\circ} \mathrm{C}$, respectively. The process itself was kept up for $4 \mathrm{~h}$. In order to unravel the growth mechanism of the NSs, other process times ranging from $1 \mathrm{~h}$ to $6 \mathrm{~h}$ have also been used. A scanning electron microscope (SEM, Zeiss Ultra 55) was used to obtain the plane and cross-sectional microstructure of the samples. X-ray diffraction (XRD, PW3040 X'Pert MPD) measurements were performed in order to obtain phase and orientation information of the NSs. Investigation by transmission electron microscopy (TEM) was carried out on Philips CM20 and FEI Titan 80-300 to acquire cross-sectional information of the NSs.

1. Schliehe, C. et al. Ultrathin $\mathrm{PbS}$ sheets by two-dimensional oriented attachment. Science 329, 550-553 (2010).

2. Ithurria, S. et al. Colloidal nanoplatelets with two-dimensional electronic structure. Nat. Mater. 10, 936-941 (2011).

3. Yu, T., Lim, B. \& Xia, Y. Aqueous-phase synthesis of single-crystal ceria nanosheets. Angew. Chem. Int. Ed. 49, 4484-4487 (2010).

4. Aagesen, M. et al. Molecular beam epitaxy growth of free-standing plane-parallel InAs nanoplates. Nat. Nanotechnol. 2, 761-764 (2007).

5. Li, X. et al. Highly conducting graphene sheets and Langmuir-Blodgett films. Nat. Nanotechnol. 3, 538-542 (2008).

6. Viswanath, B., Kundu, P., Mukherjee, B. \& Ravishankar, N. Predicting the growth of two-dimensional nanostructures. Nanotechnology 19, 195603 (2008).

7. Ali Umar, A., Oyama, M., Mat Salleh, M. \& Yeop Majlis, B. Formation of highly thin, electron-transparent gold nanoplates from nanoseeds in ternary mixtures of cetyltrimethylammonium bromide, poly(vinyl pyrrolidone), and poly(ethylene glycol). Cryst. Growth Des. 10, 3694-3698 (2010).

8. He, X., Zhao, X., Li, Y. \& Sui, X. Shape-controlled synthesis for silver: Triangular/ hexagonal nanoplates, chain-like nanoplate assemblies, and nanobelts. J. Mater. Res. 24, 2200-2209 (2009).

9. Cao, B. \& Cai, W. From $\mathrm{ZnO}$ nanorods to nanoplates: chemical bath deposition growth and surface-related emissions. The Journal of Physical Chemistry C 112, 680-685 (2007).
10. Lee, G. W. et al. Stereospecific growth of densely populated rutile mesoporous $\mathrm{TiO}_{2}$ nanoplate films: a facile low temperature chemical synthesis approach. Nanotechnology 21, 105603 (2010).

11. Huo, Z. et al. Self-organized ultrathin oxide nanocrystals. Nano Lett. 9, 1260-1264 (2009).

12. Li, C., Huang, L., Snigdha, G. P., Yu, Y. \& Cao, L. Role of boundary layer diffusion in vapor deposition growth of chalcogenide nanosheets: The case of GeS. ACS Nano 6, 8868-8877 (2012).

13. Zhao, Z., Geng, F., Cong, H., Bai, J. \& Cheng, H.-M. A simple solution route to controlled synthesis of $\mathrm{ZnS}$ submicrospheres, nanosheets and nanorods. Nanotechnology 17, 4731-4735 (2006).

14. Mattevi, C., Kim, H. \& Chhowalla, M. A review of chemical vapour deposition of graphene on copper. J. Mater. Chem. 21, 3324-3334 (2011).

15. Gan, X. et al. $\mathrm{TiO}_{2}$ nanorod-derived synthesis of upstanding hexagonal kassite nanosheet arrays: an intermediate route to novel nanoporous $\mathrm{TiO}_{2}$ nanosheet arrays. Cryst. Growth Des. 12, 289-296 (2011).

16. Zhong, H. et al. Vertically aligned graphene-like $\mathrm{SnS}_{2}$ ultrathin nanosheet arrays: excellent energy storage, catalysis, photoconduction, and field-Emitting Performances. J. Phys. Chem. C 116, 9319-9326 (2012).

17. Hosono, E., Fujihara, S., Honna, I. \& Zhou, H. S. The fabrication of an uprightstanding zinc oxide nanosheet for use in dye-sensitized solar cells. Adv. Mater. 17, 2091-2094 (2005).

18. Hsu, Y. K., Chen, Y. C., Lin, Y. G., Chen, L. C. \& Chen, K. H. Birnessite-type manganese oxides nanosheets with hole acceptor assisted photoelectrochemical activity in response to visible light. J. Mater. Chem. 22, 2733-2739 (2012).

19. Ahmad, U. \& Hahn, Y. B. ZnO nanosheet networks and hexagonal nanodiscs grown on silicon substrate: growth mechanism and structural and optical properties. Nanotechnology 17, 2174-2180 (2006).

20. Shin, J. C., Zhang, C. \& Li, X. Sub-100 nm Si nanowire and nano-sheet array formation by MacEtch using a non-lithographic InAs nanowire mask. Nanotechnology 23, 305305 (2012).

21. Cao, G. Nanostructures and Nanomaterials: Synthesis, Properties, and Applications. (Imperial College Press, London, 2004).

22. Jang, E. S., Won, J. H., Kim, Y. W., Chen, X. \& Choy, J. H. Soft-solution route to various $\mathrm{ZnO}$ nanoplate arrays. CrystEngComm 12, 3467-3470 (2010).

23. $\mathrm{Hu}, \mathrm{M}$. S. et al. The production of $\mathrm{SiC}$ nanowalls sheathed with a few layers of strained graphene and their use in heterogeneous catalysis and sensing applications. Carbon 49, 4911-4919 (2011).

24. O’Neill, A., Khan, U. \& Coleman, J. N. Preparation of high concentration dispersions of exfoliated $\mathrm{MoS}_{2}$ with increased flake size. Chem. Mater. 24, 2414-2421 (2012).

25. Wang, W. L., Wang, S. M., Cho, S. Y. \& Chang, L. Fabrication and structural property of diamond nano-platelet arrays on $\{111\}$ textured diamond film. Diamond Relat. Mater. 25, 155-158 (2012).

26. Dove, P. M., De Yoreo, J. J. \& Weiner, S. Biomineralization. Vol. 54, Reviews in Mineralogy and Geochemistry (The Mineralogical Society of America, Washington, D. C., 2003).

27. Hamilton, D. R. \& Seidensticker, R. G. Propagation mechanism of germanium dendrites. J. Appl. Phys. 31, 1165-1168 (1960).

28. Li, H., Peng, X. D. \& Ming, N. B. Comparison among the growth mechanisms of stacking-fault, twin lamella and screw dislocation - a Monte-Carlo simulation. J. Cryst. Growth 149, 241-245 (1995).

29. Ming, N., Tsukamoto, K., Sunagawa, I. \& Chernov, A. A. Stacking-faults as selfperpetuating step sources. J. Cryst. Growth 91, 11-19 (1988).

30. Ming, N. B. Defect mechanisms of crystal-growth and their kinetics. J. Cryst. Growth 128, 104-112 (1993).

31. Bogels, G., Meekes, H., Bennema, P. \& Bollen, D. Growth mechanism of vaporgrown silver crystals: Relation between twin formation and morphology. J. Phys Chem. B 103, 7577-7583 (1999).

32. Germain, V., Li, J., Ingert, D., Wang, Z. L. \& Pileni, M. P. Stacking faults in formation of silver nanodisks. J. Phys. Chem. B 107, 8717-8720 (2003).

33. Wang, C. et al. Synthesis of High-Yield Gold Nanoplates: Fast Growth Assistant with Binary Surfactants. J. Nanomater. 2010, 969030 (2010).

34. Chen, H. C. \& Chang, L. Structural investigation of diamond nanoplatelets grown by microwave plasma-enhanced chemical vapor deposition. J. Mater. Res. 20, 703-711 (2005).

35. Van Renterghem, W. et al. A TEM study of non-parallel twins inducing thickness growth in silver chloride $\{111\}$ tabular crystals. J. Imaging Sci. Technol. 45, 83-90 (2001).

36. Yin, A. X. et al. Ru nanocrystals with shape-dependent surface-enhanced Raman spectra and catalytic properties: controlled synthesis and DFT calculations. J. Am. Chem. Soc. 134, 20479-20489 (2012).

37. Sagar, A., Lee, E. J. H., Balasubramanian, K., Burghard, M. \& Kern, K. Effect of stacking order on the electric-field induced carrier modulation in graphene bilayers. Nano Lett. 9, 3124-3128 (2009).

38. Melinon, P., Masenelli, B., Tournus, F. \& Perez, A. Playing with carbon and silicon at the nanoscale. Nat. Mater. 6, 479-490 (2007).

39. Yagi, K. \& Nagasawa, H. Crystallinity of 3C-SiC films grown on Si substrates. Mater. Sci. Forum 264-268, 191-194 (1998).

40. Nagasawa, H., Yagi, K., Kawahara, T. \& Hatta, N. Reducing planar defects in 3CSiC. Chem. Vap. Deposition 12, 502-508 (2006). 
41. Nagasawa, H., Yagi, K. \& Kawahara, T. 3C-SiC hetero-epitaxial growth on undulant $\mathrm{Si}(001)$ substrate. J. Cryst. Growth 237-239, (Part 2), 1244-1249 (2002).

42. Levchenko, I. \& Ostrikov, K. Nanostructures of various dimensionalities from plasma and neutral fluxes. J. Phys. D: Appl. Phys. 40, 2308-2319 (2007).

43. Tsuboi, H. et al. Computational evaluation of electrical conductivity on $\mathrm{SiC}$ and the influence of crystal defects. Mater. Sci. Forum 600-603, 497-500 (2009).

44. Song, X. et al. Evidence of electrical activity of extended defects in 3C-SiC grown on Si. Appl. Phys. Lett. 96, 142104 (2010).

45. Jiang, X. \& Jia, C. L. Direct local epitaxy of diamond on $\mathrm{Si}(100)$ and surfaceroughening-induced crystal misorientation. Phys. Rev. Lett. 84, 3658-3661 (2000).

46. Fukuda, K. et al. Electron-cyclotron-resonance plasma-enhanced chemical vapor deposition of epitaxial Si without substrate heating by ultraclean processing. Appl. Phys. Lett. 59, 2853-2855 (1991).

47. Li, B. S. et al. High quality $\mathrm{ZnO}$ thin films grown by plasma enhanced chemical vapor deposition. J. Appl. Phys. 91, 501-505 (2002).

48. Zhang, W. J. et al. The mechanism of chemical vapor deposition of cubic boron nitride films from fluorine-containing species. Angew. Chem., Int. Ed. 44, 4749-4753 (2005)

49. Sun, L. et al. Electronic structures of SiC nanoribbons. J. Chem. Phys. 129, 174114 (2008).

50. Xu, B., Yin, J., Xia, Y. D., Wan, X. G. \& Liu, Z. G. Ferromagnetic and antiferromagnetic properties of the semihydrogenated SiC sheet. Appl. Phys. Lett. 96, $143111(2010)$

51. Lou, P. \& Lee, J. Y. Band structures of narrow zigzag silicon carbon nanoribbons. J. Phys. Chem. C 113, 12637-12640 (2009).

52. Hsueh, H. C., Guo, G. Y. \& Louie, S. G. Excitonic effects in the optical properties of a SiC sheet and nanotubes. Phys. Rev. B 84, 085404 (2011).

\section{Acknowledgements}

The authors would like to thank Chun-Lin Jia, Peter Grünberg Institute and Ernst Ruska-Centre (ER-C) for Microscopy and Spectroscopy with Electrons,

Forschungszentrum Jülich, and Nianjun Yang, Fraunhofer Institue IAF, for stimulating discussions. The authors also would like to thank Kun Chen, department of Organic Chemistry I, University of Siegen for his help in electrochemical characterization.

\section{Author contributions}

H. Zhuang, L. Zhang, T. Staedler, and X. Jiang proposed the experiment and planned the research. H. Zhuang synthesized the nanosheets and performed the SEM analysis. L. Zhang performed systematic TEM analysis. R. Fuchs performed AFM analysis. T. Staedler and X. Jiang supervised the project. H. Zhuang prepared the manuscript with input from all authors.

\section{Additional information}

Supplementary information accompanies this paper at http://www.nature.com/ scientificreports

Competing financial interests: The authors declare no competing financial interests. How to cite this article: Zhuang, H., Zhang, L., Fuchs, R., Staedler, T. \& Jiang, X. When epitaxy meets plasma: a path to ordered nanosheets arrays. Sci. Rep. 3, 2427; DOI:10.1038/ srep02427 (2013).

\footnotetext{
(c) (1) This work is licensed under a Creative Commons Attribution-

BY NG SA NonCommercial-ShareAlike 3.0 Unported license. To view a copy of this license, visit http://creativecommons.org/licenses/by-nc-sa/3.0
} 